\title{
Perfil epidemiológico dos casos de tuberculose drogarresistente
}

\author{
Epidemiological profile of drug-resistant tuberculosis cases
}

Perfil epidemiológico de los casos de tuberculosis farmacorresistente

Ícaro Moraes de Oliveira Valença ${ }^{1}$, Morgana Cristina Leôncio de Lima ${ }^{\star}$, Cynthia Angélica Ramos de Oliveira Dourado², Maria Sandra Andrade ${ }^{2}$, Ana Cristina Nóbrega Silva Falcão ${ }^{1}$, Wânia Maria de Sá Pereira ${ }^{1}$, Isabelle Andrian Pereira da Silva ${ }^{1}$, Alexandra Barros de Santana ${ }^{1}$, Andréa Alexandre Valença Moraes ${ }^{3}$, Clarissa Mourão Pinho².

\section{RESUMO}

Objetivo: Descrever o perfil epidemiológico de pacientes com tuberculose drogarresistente. Métodos: Tratase de estudo descritivo, transversal, com abordagem quantitativa, o qual realizou-se por meio de notificações do Sistema de Informação de Tratamento Especiais de Tuberculose no período de 2014 a 2019. Através da busca de 60 prontuários de pacientes notificados com resistência, os quais obtiveram o término dos seus respectivos tratamentos em um hospital de referência no tratamento de tuberculose drogarresistente do estado de Pernambuco. Resultados: A amostra compôs-se por 60 pacientes, com predominância do sexo masculino e com média de idade de 45,12 anos. Desse modo, observou-se que a prevalência dos multirresistentes, resolutos a rifampicina e a isoniazida, por seguinte, deu-se como cura ao fim do tratamento. Conclusão: Verifica-se a necessidade da adoção do Tratamento Diretamente Observado e o cuidado compartilhado com Atenção Primária à Saúde com vistas ao aumento da adesão. Além disso, faz necessário o aprimoramento da vigilância epidemiológica com objetivo de obter dados mais consistentes.

Palavras-chave: Tuberculose extensivamente resistente a medicamentos, Perfil de saúde, Tuberculose resistente a múltiplos medicamentos.

\begin{abstract}
Objective: Describe the epidemiological profile of patients withdrug-resistant tuberculosis. Methods: This is a descriptive, cross-sectional study with a quantitative approach, which was carried out through notifications fromthe Special Treatment Information System for Tuberculosis in the period from 2014 to 2019. Through the search of 60 records of patients notified with resistance, who reached theend of their respective treatments in a referral hospital for the treatment of drug-resistant tuberculosis in the state of Pernambuco. Results: The sample consisted of 60 patients, predominantly male and with anaverage age of 45.12 years. Thus, it was observed that the prevalence of multidrug-resistant, resolute rifampicin and isoniazid, as a result, occurred as a cure attheend of treatment. Conclusion: There is a need for the adoption of the Directly Observed Treatment and shared care with Primary Health Care in or der to increasead herence. In addition, it is necessary to improve epidemiological surveillance in ordertoobtain more consistent data.
\end{abstract}

Keywords: Extensively drug-resistant tuberculosis, Health profile, Multidrug-resistant tuberculosis.

\section{RESUMEN}

Objetivo: Describirel perfil epidemiológico de pacientes com tuberculosis farmacorresistente. Métodos: Este es um estúdio descriptivo, transversal com un enfoque cuantitativo, que se realizó mediante notificaciones del Sistema de Información de Tratamiento Especial para La Tuberculosis em el período de 2014 a 2019. A través de La búsqueda de 60 registros de pacientes notificados con resistencia, quienes obtuvieron el final de sus

\footnotetext{
${ }^{1}$ Secretaria Estadual de Saúde do Estado de Pernambuco, Recife - PE.

*E-mail: limamorgana124@gmail.com

2Universidade de Pernambuco (UPE), Recife - PE.

${ }^{3}$ Centro Universitário Leonardo da Vinci (UNIASSELVI). Rio de Janeiro - RJ.
} 
respectivos tratamientos en un hospital de referencia para El tratamiento de La tuberculosis farmacorresistente en el estado de Pernambuco. Resultados: La muestra consistió en 60 pacientes, predominantemente hombres y con una edad promedio de 45,12 años. Por lo tanto, se observó que La prevalencia de rifampicina e isoniazida resueltas y resistentes a múltiples fármacos, para El siguiente, ocurrió como una cura al final del tratamiento. Conclusión: Es necesario adoptar un tratamiento directamente observado y una atención compartida con La atención primaria de salud con miras a aumentar La adherencia. Además, es necesario mejorar La vigilancia epidemiológica para obtener datos más consistentes.

Palabras clave: Tuberculosis extensivamente resistente a drogas, Perfil de salud, Tuberculosis resistente a múltiples medicamentos.

\section{INTRODUÇÃO}

A Tuberculose (TB) é uma doença infectocontagiosa, prevenível e curável, cujo agente etiológico é o Mycobacterium tuberculosis, conhecido também como bacilo de Koch. Devido ao aumento gradativo do número de casos nos últimos anos a doença é considerada um problema de saúde pública (MORAES MFV, et al., 2017; OLIVEIRA LB, et al., 2018). A incidência da TB está fortemente associada às comorbidades, como: HIV, Aids, tabagismo, etilismo, desnutrição e diabetes mellitus (SANTOS TA e MARTINS MMF, 2018).

Considerada uma doença negligenciada, a TB corresponde a um grupo de enfermidades infectocontagiosas com características de endemicidade que acometem principalmente as populações mais vulneráveis. Guarda relação com desigualdade social, pobreza, diminuição da produtividade da classe trabalhadora e redução da qualidade de vida das pessoas, refletindo as iniquidades sociais em saúde. Sendo assim, a ocorrência da tuberculose tem implicações sociais e epidemiológicas, o que dificulta seu controle e prevenção (SANTOS ML, et al., 2018).

Atualmente tem se observado que fatores como o diagnóstico precoce, e tratamento oportuno são consideradas as principais medidas de controle da epidemia. Sendo determinantes no prognóstico e diminuição das taxas de mortalidade da infecção. Em contrapartida o aumento no número de tuberculose drogarresistente (TB-DR), surge principalmente devido à dificuldade da adesão terapêutica e sucessivos abandonos caracterizando fator de impacto negativo na situação epidemiológica em diversos países. (FERREIRA DP, 2019; SANTOS TA e MARTINS MMF, 2018; CAMINERO JA, et al., 2017).

Dados da Organização Mundial de Saúde (OMS), revelam que em 2016, houve 1,67 milhão de óbitos por TB. Neste mesmo ano, foram registrados cerca de 490 mil casos de tuberculose multirresistente (TB-MDR), destes $6,2 \%$ dos casos foram associados à infecção por cepas de Tuberculose Extensivamente Resistentes (TB-XDR). Estima-se que 3,9 dos novos casos de TB diagnosticados no mundo sejam Tuberculose Mulrresistentes (TB-MDR). A TB-MDR é definida como resistência a pelo menos isoniazida e rifampicina, os dois medicamentos anti-TB de primeira linha mais eficazes (WHO, 2016).

Segundo as últimas recomendações do Ministério da saúde (2019) classifica tuberculose drogarresistente em seis tipos de resistência, sendo elas: Monorresistente (resistência a somente um fármaco antituberculose); Polirresistência (resistência a dois ou mais fármacos antituberculose, exceto associação rifampicina e isoniazida); Multirresistente (resistência a pelo menos à rifampicina e isoniazida); Resistência extensiva (resistência à pelo a rifampicina e isoniazida, acrescida a resistência a fluorioquinolona e a um injetável de segunda linha - amicacina, canamicina ou capreomicina) e Resistência à rifampicina (resistência à rifampicina identificada por meio do TRM-TB exclusivamente), ainda sem outras resistências conhecidas).

O tratamento da TB-DR à rifampicina, de modo isolado ou combinado, trata-se de um grande desafio para o tratamento a doença. Esquemas sem rifampicina requerem o uso de fármacos de segunda linha, resultando em tratamentos mais prolongados, com maior risco de toxicidade e pior prognóstico (CAMINERO JA, et al., 2017).

Para tais casos, o Ministério da Saúde recomenda o referenciamento para unidades terciárias de atenção a pessoas com TB, devido à complexidade do tratamento. Destaca-se a importância do compartilhamento do cuidado com a Atenção Primária à Saúde com o objetivo de oferecer o Tratamento Diretamente Observado (TDO), favorecendo assim, a adesão e cura dos casos (BRASIL, 2019). 
Com base na literatura exposta, torna-se relevante a investigação do perfil epidemiológico dos casos de TB-DR, uma vez que apesar de todos os avanços na medicina e na indústria farmacêutica, a TB ainda apresenta uma elevada incidência e embora seja uma doença tratável e curável, esta é responsável por um alto número de óbitos. Ainda, muitos destes casos evoluem para resistência às drogas, dificultando o tratamento e proporcionando a proliferação de bacilos cada vez mais resistentes. Diante do exposto, o presente estudo tem como objetivo descrever o perfil epidemiológico dos pacientes com tuberculose drogarresistente.

\section{MÉTODOS}

A pesquisa realizada trata-se de um estudo transversal, descritivo, com abordagem quantitativa, realizado em uma unidade de referência no tratamento de TB-DR do estado de Pernambuco. A coleta de dados foi realizada nos meses referentes a abril e a maio de 2020.

Nessas circunstâncias, a população formou-se por indivíduos diagnosticados com algum tipo de TB resistente, no período compreendido entre os anos de 2014 e 2019, que tenham concluído o tratamento. Dessa forma, justifica-se a necessidade da conclusão do tratamento para que seja obtido conhecimento em torno da condução do tratamento, bem como seu desfecho.

Diante disso, realizou-se um levantamento quantitativo de todos os casos notificados pela unidade, com pelo menos resistência a uma droga, os quais encontravam-se na fase de acompanhamento em relação a tuberculose drogarresistente, isso é: a) $\geq 1$ ano e 6 meses de tratamento até 5 anos; b) com faixa etária $\geq 18$ anos de idade; c) de ambos os sexos; d) notificados no período de 2014 a 2019. Nesse sentido, identificouse 340 notificações, contudo, apenas 60 correspondentes aos critérios de elegibilidade. Para a condução da coleta dos dados foi levado em consideração à ficha de notificação do Sistema de Informação de Tratamento Especial de Tuberculose (SITE-TB) do Ministério da Saúde (MS) (BRASIL, 2017).

A ficha investiga questões voltadas a dados sociodemográficos, como sexo, idade, cor, gestação, ocupação, escolaridade, dados do caso - comorbidades, local de contágio, tipo de TB-DR, resistência, forma clínica, tratamento atual optando por esquema padronizado ou individualizado, exames complementares baciloscopia, cultura, biologia molecular, teste de sensibilidade e HIV iniciais, tratamentos anteriores - número de tratamentos anteriores, tratamentos adequados, resultado dos trabalhos anteriores e tipo de resistência.

Nessa perspectiva, o armazenamento e a tabulação dos dados efetuaram-se através do software Microsoft Excel 2013. Por meio de uma análise posterior, os dados foram perscrutados com o auxílio do programa estatístico SPSS versão 20.0. Assim, avaliou-se a distribuição das variáveis quantitativas categóricas, calculando frequências absolutas e percentuais das referidas variáveis, apresentadas por meio de tabelas simples e do tipo de contingência, a qual evidencia a distribuição de uma variável em relação a outra. Buscando obter a análise da distribuição das variáveis quantitativas, considerou-se a medida de tendência central, média e a dispersão desvio padrão, assim como o intervalo mínimo e máximo. Para isso, utilizou-se o teste de qui-quadrado (X2) para comparação de proporções, considerando o nível de significância de 5,0\% $(\mathrm{p}<0,05)$.

Foram respeitados os preceitos éticos da Resolução no 466/2012 do Conselho Nacional de Saúde do Ministério da Saúde, referente a estudos com seres humanos e a pesquisa foi aprovada pelo Comitê de Ética e Pesquisa do Hospital Otávio de Freitas (HOF), com parecer: 3.737.733.

\section{RESULTADOS}

Por meio dos resultados desta pesquisa, evidencia-se o perfil de 60 pacientes com TB-DR, sendo estes, notificados e acompanhados por uma unidade de referência na cidade de Recife, Pernambuco. Reitera-se que $o$ ano de diagnóstico variou entre os anos de 2010 e 2016, sendo 2 pacientes (3,3\%) diagnosticados em 2010, 3 (5,0\%) em 2011, 24 (40,0\%) em 2012, 9 (15,0\%) em 2013, 17 (28,3\%) em 2014, 4 (6,7\%) em 2015 e $1(1,7 \%)$ em 2016. Acerca do ano de início do tratamento, também se verificou variações entre os anos de 2010 e 2016, sendo 2 (3,3) em 2010, 18 (30,0\%) em 2012, 15 (25,0\%) em 2013, 21 (35,0\%) em 2014, 2 (3,3\%) em 2015 e 2 (3,3\%) em 2016. 
Tomando como base os respectivos dados, verifica-se a predominância do sexo masculino, com faixa etária entre 36 e 50 anos, apresentando entre 4 a 7 anos de estudo. Acerca da variável "ocupação", 95\% dos casos foram ignorados. Dessa forma, os resultados do teste Qui-quadrado para comparação de proporção, evidenciam a prevalência dos fatores avaliativos, indicando que o grupo predominante se tornou significativamente maior que os demais, exceto, a escolaridade, comorbidade e a procedência, o que indica uma distribuição homogênea entre os grupos de cada fator (Tabela 1).

Tabela 1 - Distribuição dos dados sociodemográficos dos casos de tuberculose drogarresistente em um Hospital Referência, 2020.

\begin{tabular}{|c|c|c|c|}
\hline Fator avaliado & $\mathbf{n}$ & $\%$ & p-valor ${ }^{1}$ \\
\hline \multicolumn{4}{|l|}{ Sexo } \\
\hline Masculino & 42 & 70 & \multirow{2}{*}{$<0,001$} \\
\hline Feminino & 18 & 30 & \\
\hline \multicolumn{4}{|l|}{ Idade em anos } \\
\hline Até 35 & 17 & 28,3 & \multirow{5}{*}{$<0,001$} \\
\hline 36 a 50 & 27 & 45 & \\
\hline$>50$ & 16 & 26,7 & \\
\hline Mínimo - Máximo & & & \\
\hline Média \pm Desvio padrão & \multicolumn{2}{|c|}{$42,15 \pm 10,9$} & \\
\hline \multicolumn{4}{|l|}{ Escolaridade } \\
\hline Nenhuma & 2 & 3,3 & \multirow{6}{*}{0,067} \\
\hline de 1 a 3 anos & 13 & 21,7 & \\
\hline de 4 a 7 anos & 21 & 35 & \\
\hline de 8 a 11 anos & 16 & 26,7 & \\
\hline 12 ou mais & 4 & 6,7 & \\
\hline Ignorado & 4 & 6,7 & \\
\hline \multicolumn{4}{|l|}{ Procedência } \\
\hline Recife & 23 & 38,3 & \multirow{3}{*}{0,076} \\
\hline Região Metropolitana do Recife & 26 & 43,3 & \\
\hline Interior do estado & 11 & 18,3 & \\
\hline \multicolumn{4}{|l|}{ Raça/Cor } \\
\hline Branca & 10 & 16,7 & \multirow{3}{*}{$<0,001$} \\
\hline Negra & 1 & 1,7 & \\
\hline Parda & 49 & 81,7 & \\
\hline \multicolumn{4}{|l|}{ Ocupação } \\
\hline Profissional do sistema penitenciário & 1 & 1,7 & \multirow{3}{*}{$<0,001$} \\
\hline Ambulante & 2 & 3,3 & \\
\hline Ignorado & 57 & 95 & \\
\hline \multicolumn{4}{|l|}{ Comorbidades } \\
\hline Nenhuma & 29 & 48,3 & \multirow{9}{*}{0,004} \\
\hline AIDS & 3 & 5 & \\
\hline Alcoolismo & 3 & 5 & \\
\hline Diabetes & 10 & 16,7 & \\
\hline Tabagismo & 4 & 6,7 & \\
\hline Usuário de drogas ilícitas & 1 & 1,7 & \\
\hline AIDS + diabetes & 3 & 5 & \\
\hline Alcoolismo + tabagismo & 6 & 10 & \\
\hline Alcoolismo + diabetes & 1 & 1,7 & \\
\hline
\end{tabular}

Legenda: ${ }^{1} p$-valor do teste Qui-quadrado para comparação de proporção.

Fonte: VALENÇA IMO, et al., 2020. 
Na tabela 2 observa-se dados incógnitos acerca do local de contágio, padrão de multirresistência da forma pulmonar em casos novos da doença e com apresentação radiológica clássica da TB, a forma cavitária. Todavia, percebe-se que, a maioria dos casos foram classificados como "Novo TB-DR", seguido daqueles com falência ao primeiro tratamento para TB-DR. Na tabela 2, observa-se muitos dados ignorados acerca do local de contágio, um padrão.

Tabela 2 - Caracterização do perfil clínico dos casos de tuberculose drogarresistente em um Hospital de Referência, 2020.

\begin{tabular}{|c|c|c|}
\hline Fator avaliado & $\mathbf{n}$ & $\%$ \\
\hline \multicolumn{3}{|l|}{ Local provável de contágio } \\
\hline Instituição prisional & 2 & 3,3 \\
\hline Ignorado & 58 & 96,7 \\
\hline \multicolumn{3}{|l|}{ Padrão de resistência } \\
\hline Monorresistência & 9 & 15 \\
\hline Polirresistência & 1 & 1,7 \\
\hline Mutirresistência & 50 & 83,3 \\
\hline \multicolumn{3}{|l|}{ Formas Clínicas } \\
\hline Pulmonar & 59 & 98,3 \\
\hline Pulmonar + extrapulmonar & 1 & 1,7 \\
\hline \multicolumn{3}{|c|}{ Tipo Entrada de tuberculose drogarresistente. } \\
\hline Caso Novo & 25 & 41,7 \\
\hline Reingresso Após Abandono & 7 & 11,7 \\
\hline Recidiva & 9 & 15 \\
\hline Falência ao primeiro tratamento & 11 & 18,3 \\
\hline Falência de retratamento & 7 & 11,7 \\
\hline Mudança do padrão de resistência & 1 & 1,7 \\
\hline \multicolumn{3}{|l|}{ Pulmonar } \\
\hline Unilateral cavitária & 22 & 36,7 \\
\hline Unilateral não cavitária & 1 & 1,7 \\
\hline Bilateral cavitária & 36 & 60 \\
\hline Bilateral não cavitária & 1 & 1,7 \\
\hline \multicolumn{3}{|l|}{ Peso inicial } \\
\hline Mínimo - Máximo & \multicolumn{2}{|c|}{$31-100$} \\
\hline Média \pm Desvio padrão & \multicolumn{2}{|c|}{$56,6 \pm 15,9$} \\
\hline \multicolumn{3}{|l|}{ Peso final } \\
\hline Mínimo - Máximo & \multicolumn{2}{|c|}{$31,4-100$} \\
\hline Média \pm Desvio padrão & \multicolumn{2}{|c|}{$61,5 \pm 16,4$} \\
\hline
\end{tabular}

Fonte: VALENÇA IMO, et al., 2020.

Diante dos dados denotados, cabe observar a referente distribuição relacionada aos tratamentos dos casos notificados. Sendo assim, $26,7 \%$ apresentaram baciloscopia inicial positiva, destaca-se que, acerca da baciloscopia final, $65 \%$ da amostra não realizou esse exame complementar, o que pode ser justificado pela redução ou escassez de escarro para realização do exame. Os dados revelam que a maioria dos casos se apresentou como esquema padronizado para multirresistência $4(40 \%)$ e resistência a rifampicina e isoniazida $(38,3 \%)$ (Tabela 3).

Acerca do exame para HIV, verifica-se que a maioria teve resultado negativo, sendo $61,7 \%$. Contudo, observa-se que $31,7 \%$ dos casos não realizaram o exame complementar. Em relação aos tratamentos anteriores, cerca de metade dos casos haviam realizado dois ou mais tratamentos. Por fim, os resultados revelam dados positivos, uma vez que $60 \%$ dos casos obtiveram a cura, no entanto, o número de abandonos é alto com índice de $25 \%$ (Tabela 3). 
Tabela 3 - Distribuição dos dados laboratoriais e de tratamento dos casos de tuberculose drogarresistente em uma unidade de referência, 2020.

\begin{tabular}{|c|c|c|}
\hline Fator avaliado & $\mathbf{n}$ & $\%$ \\
\hline \multicolumn{3}{|l|}{ Esquema padronizado } \\
\hline Básico & 1 & 1,7 \\
\hline Hepatologia 3 & 1 & 1,7 \\
\hline Hepatoxicidade ou intolerância a isoniazida & 1 & 1,7 \\
\hline Monorresistência a isoniazida & 7 & 11,7 \\
\hline Multirresistência 1 & 8 & 13,3 \\
\hline Multirresistência 2 & 18 & 30 \\
\hline Multirresistência 4 & 24 & 40 \\
\hline \multicolumn{3}{|l|}{ Resistente a: [CD2] } \\
\hline Amicacina+ofloxacina+Canamicina & 1 & 1,7 \\
\hline Etambutol+estreptomicina & 1 & 1,7 \\
\hline Isoniazida & 9 & 15 \\
\hline Isoniazida+ estreptomicina & 2 & 3,3 \\
\hline Isoniazida+pirazinamida & 1 & 1,7 \\
\hline Rifampicina+ etambutol+ estreptomicina & 1 & 1,7 \\
\hline Rifampicina + isoniazida & 23 & 38,3 \\
\hline Rifampicina+isoniazida+etambutol & 10 & 16,7 \\
\hline Rifampicina+isoniazida+etambutol+estreptomicina & 2 & 3,4 \\
\hline Rifampicina+isoniazida+etambutol+pirazinamida+estreptomicina+moxifloxacino & $\overline{1}$ & 1,7 \\
\hline Rifampicina+isoniazida+ estreptomicina & 8 & 13,3 \\
\hline Rifampicina+Isoniazida & 1 & 1,7 \\
\hline \multicolumn{3}{|l|}{ Sensível a: } \\
\hline Não há sensibilidade & 10 & 16,7 \\
\hline Rifampicina & 1 & 1,7 \\
\hline Isoniazida & 1 & 1,7 \\
\hline Amicacina+capreomicina+Canamicina & 1 & 1,7 \\
\hline Amicacina+capreomicina+ofloxacina+ Canamicina & 1 & 1,7 \\
\hline Amicacina+capreomicina+ofloxacina+Canamicina+ moxifloxacino & 1 & 1,7 \\
\hline Capreomicina & 1 & 1,7 \\
\hline Etambutol & 1 & 1,7 \\
\hline Etambutol+amicacina+capreomicina+ofloxacina+Canamicina & 1 & 1,7 \\
\hline Etambutol+ estreptomicina & 20 & 33,3 \\
\hline Etambutol+ estreptomicina+rifampicina & 1 & 1,7 \\
\hline Etambutol+ estreptomicina & 1 & 1,7 \\
\hline Rifampicina+etambutol & 2 & 3,3 \\
\hline Rifampicina+etambutol+ estreptomicina & 9 & 15 \\
\hline Rifampicina+ isoniazida & 1 & 1,7 \\
\hline Estreptomicina & 6 & 10 \\
\hline Pirazinamida + estreptomicina & 1 & 1,7 \\
\hline \multicolumn{3}{|l|}{ Baciloscopia Inicial } \\
\hline Negativa & 5 & 8,3 \\
\hline Positiva (sem + ) & 16 & 26,7 \\
\hline & 9 & 15 \\
\hline$(++)$ & 13 & 21,7 \\
\hline$(+++)$ & 17 & 28,3 \\
\hline \multicolumn{3}{|l|}{ Baciloscopia Final } \\
\hline Não realizada & 39 & 65 \\
\hline Negativa & 6 & 10 \\
\hline Positiva & 6 & 10 \\
\hline$(+)$ & 1 & 1,7 \\
\hline$(++)$ & 7 & 11,7 \\
\hline$(+++)$ & 1 & 1,7 \\
\hline \multicolumn{3}{|l|}{ Tipo de Resistência } \\
\hline Primária & 5 & 8,3 \\
\hline Adquirida & 55 & 91,7 \\
\hline \multicolumn{3}{|l|}{ Teste HIV } \\
\hline Positivo & 4 & 6,7 \\
\hline Negativo & 37 & 61,7 \\
\hline Não realizou & 19 & 31,7 \\
\hline \multicolumn{3}{|l|}{ Número Tratamento anterior } \\
\hline Nenhum tratamento anterior & 11 & 18,3 \\
\hline 1 tratamento anterior & 22 & 36,7 \\
\hline 2 ou mais tratamentos anteriores & 27 & 45 \\
\hline \multicolumn{3}{|l|}{ Desfecho } \\
\hline $\begin{array}{l}\text { Cura } \\
\text { Abandono }\end{array}$ & $\begin{array}{l}36 \\
15\end{array}$ & $\begin{array}{l}60 \\
25\end{array}$ \\
\hline Óbito & 9 & 15 \\
\hline
\end{tabular}

Fonte: VALENÇA IMO, et al., 2020. 
Identifica-se que $76 \%$ da amostra de novo caso de TB-DR cursaram com a remissão da doença, com resultado de óbito. Assim, as maiores prevalências encontram-se nos casos de pessoas as quais tornaramse TB-DR devido ao abandono do tratamento para resistência, nos casos de falência no primeiro tratamento de TB-DR e falência de retratamento da TB-DR (Tabela 4).

Tabela 4 - Distribuição dos tipos de tuberculose drogarresistente segundo os desfechos apresentados dos casos de TBDR em uma unidade de referência, 2020.

\begin{tabular}{lccc}
\hline \multirow{2}{*}{ Fator avaliado } & \multicolumn{3}{c}{ Desfecho } \\
\cline { 2 - 4 } & Cura & Abandono & Óbito \\
\hline Tipo de Entrada tuberculose drogarresistente. & & & \\
\hline Caso Novo & $19(76,0 \%)$ & $05(20,0 \%)$ & $01(4,0 \%)$ \\
Reingresso Após Abandono & $02(28,6 \%)$ & $03(42,9 \%)$ & $02(28,6 \%)$ \\
Recidiva & $05(55,6 \%)$ & $03(33,3 \%)$ & $01(11,1 \%)$ \\
Falência ao primeiro tratamento & $04(36,4 \%)$ & $04(36,4 \%)$ & $03(27,3 \%)$ \\
Falência de retratamento & $05(71,4 \%)$ & $00(0,0 \%)$ & $02(28,6 \%)$ \\
Mudança do padrão de resistência & $01(100,0 \%)$ & $00(0,0 \%)$ & $00(0,0 \%)$ \\
\hline
\end{tabular}

Fonte: VALENÇA IMO, et al., 2020.

\section{DISCUSSÃO}

Diante dos dados do presente estudo, permitiu-se descrever o perfil dos indivíduos acometidos pela TBDR e o desfecho clínico inerente ao processo saúde doença, o qual aponta que os pacientes se tornaram resistentes aos medicamentos e foram a óbito, abandonaram o tratamento. Por conseguinte, observou-se que $45 \%$ realizaram dois ou mais tratamentos anteriores. Desse modo, percebe-se, também, amostra constituída em sua maioria por homens adultos, faixa etária média, cor parda, com predomínio do tipo de entrada no tratamento classificada em caso novo e resultado clínico dado pela cura da doença no paciente.

Os resultados são positivos acerca da maioria dos desfechos clínicos ser a cura, uma vez que as estimativas globais apontam que apenas 1 a cada 10 pacientes com TB-MDR são tratados com sucesso. Tais dados reforçam a importância de um sistema de saúde universal, como o Sistema Único de Saúde (SUS), dado que a literatura evidencia que a maior carga de resistência a medicamentos se concentra nos continentes da Europa, Ásia e África, o que demonstra um sistema de saúde fraco e com falhas em todos os estágios do caminho ao atendimento a tal doença (GUPTA-WRIGHT A, et al., 2018).

Acerca do perfil sociodemográfico apresentado, constata-se parcela expressiva composta pelo sexo masculino, o que se pode atribuir ao fato de muitos dos homens não buscam os serviços de saúde, especialmente as ações e atividades de prevenção e promoção à saúde. Ainda, verifica-se nas políticas de saúde do homem a relação de maior exposição a diversas situações de riscos evitáveis à saúde e ao estilo de vida mais agressivo que repercute na necessidade de uma assistência e cuidado de saúde no nível terciário (BRASIL, 2018a; BRASIL, 2018b).

Estudo nacional recente corrobora com o perfil encontrado neste estudo. Percebe-se que de um total de 257 pacientes notificados, $69,6 \%$ era composto pelo sexo masculino, 52,5\% tinham idade entre 30 e 49 anos, $58 \%$ tinham menos de 8 anos de estudo e $35 \%$ se consideram pardos. Além disso, observou-se que ser homem estava relacionado ao aumento do número de abandonos e óbitos e ter menos de 8 anos de estudo foi associado ao aumento do risco de abandono do tratamento (VIANA PVS, et al., 2018). A literatura já evidencia a relação entre ao nível de escolaridade e a adesão terapêutica, uma vez que ter um maior nível de escolaridade apresenta uma maior probabilidade de aderir ao tratamento devido a uma maior compreensão e acesso a informações acerca da doença (OLIVEIRA SAG, et al., 2019).

Sobre os aspectos clínicos, nota-se uma predominância dos casos multirresistentes, com forma clínica pulmonar e com entrada no sistema de informação como caso novo TB-DR, seguido por falência de retratamento e recidiva TB-DR. Além disso, a maioria dos casos incluídos neste estudo tinham como esquema padronizado para multirresistência 4, seguido da multirresistência 2, eram resistentes a rifampicina e isoniazida, principais drogas para tratamento da tuberculose. 
Estudo desenvolvido na Alemanha identificou similarmente padrões predominantes de resistência à Rifampicina $(\mathrm{R})$ e Isoniazida $(\mathrm{H})$, contudo os dados revelaram proporções inesperadamente altas de resistências Estreptomicina (S) e H em crianças, como também alta de resistência a Pirazinamida (Z) entre a população idosa, esse achado nas crianças possivelmente pode estar associado ao desafio inerente a TB infantil paucibacilar, que pode retardar a identificação oportuna da TB e na suspeita de resistência a medicamentos (RABAHI MF, et al., 2017; GLASAUER S, et al., 2019).

No Brasil, a TB em crianças segue o esquema terapêutico com três $\mathrm{R}, \mathrm{H}$ e S, esta decisão se fundamenta no menor risco de resistência à $\mathrm{H}$ devido à pequena carga bacilar, mais comum na TB infantil. Diante 0 exposto, os resultados diferem na presente pesquisa que encontrou predomínio da resistência medicamentosa em adultos, sendo assim importante apoiar o padrão e desenho apropriado dos regimes de tratamentos, bem como a vigilância epidemiológica dos casos e estratégias de controle (RABAHI MF, et al., 2017; GLASAUER S, et al., 2019).

Os dados demonstram que a maioria dos indivíduos não realizaram baciloscopia final, que $1 / 4$ daqueles que fizeram ainda deu positiva no final do tratamento e abandonaram o tratamento. Para redução do abandono e aumento da adesão medicamentosa são mensuradas na literatura como estratégias de controle e atenção o TDO, descentralização da assistência para atenção primária, uso de ferramentas de comunicação, como software e plataformas eletrônicas, que proporcionam a consultoria médica entre especialistas e diferentes profissionais e oferta de apoio emocional, social e econômico, com incentivo financeiro, apoio alimentar, construção de moradias e apoio psicológico (BALLESTERO JGA, et al., 2019).

O TDO é recomendado pela OMS desde a década de 90, este tem como objetivo aumentar a adesão ao tratamento da TB. O TDO deve seguir cinco elementos, sendo eles: compromisso político e assistência financeiro para atividades de controle da TB; identificação dos casos de TB através de baciloscopia de sintomáticos respiratórios; esquemas padronizados e incentivo aos programas de TDO nos dois primeiros meses; garantia no fornecimento dos medicamentos tuberculostáticos; fortalecimento do sistema de notificação e avaliação dos resultados do tratamento de cada paciente, de forma individualizada e do programa de controle da tuberculose (PARIDA A, et al., 2014; RABAHI MF, et al., 2017).

Em revisão sistemática recente, com objetivo de avaliar a eficácia do tratamento descentralizado a TB-DR em comparação a abordagem centralizada, observou que o cuidado descentralizada apresenta melhores resultados que os centralizados, uma vez que se verificou o aumento da adesão medicamentosa, maior apoio familiar e social, redução de custos, diminuição das taxas de falha no tratamento e mortalidade (HO J, et al., 2017).

Ainda, verifica-se que houve a predominância daqueles com resistência adquirida, que realizaram 2 ou mais tratamentos. No que concerne os debates no tocante aos entraves do tratamento para TB, assim como a TB sensível a TB resistente tem relação com histórico de irregularidades e falência dos esquemas terapêuticos que comprometem a adesão ao tratamento. Nesse sentido, muitos pacientes possuem sucessivos tratamentos anteriores e abandonos frequentes, tendo como possíveis fatores do insucesso terapêutico: aspectos socioeconômicos, uso abusivo de álcool e outras drogas, tratamentos indisponíveis, a qualidade dos serviços de saúde, bem como fragilidade do vínculo com profissional de saúde. Tais aspectos aumentam a mortalidade por indivíduos com TB-DR (BRASIL, 2019; SOUSA, et al., 2019).

Quanto ao teste de HIV, nota-se que a maior parte obteve resultado negativo, contudo, mais de $30 \%$ da amostra não realizado o exame. É demonstrado na literatura a associação entre o HIV e a infecção por TB$\mathrm{DR}$, bem como o aumento no número de óbitos com esta associação. Nesta perspectiva, ressalta-se a importância da investigação da TB em Pessoas Vivendo com HIV, assim como, oferta da testagem para HIV a todos indivíduos diagnosticados com TB/ TB-DR e início precoce do tratamento da coinfecção, uma vez que pacientes coinfectados possuem menor taxa de sobrevida (OLIVEIRA SAG, et al., 2019; NUGRAHAENI DN e ZAQUIYA S, 2019).

Com relação a associação dos tipos de TB-DR e o desfecho, nota-se que a maioria daqueles classificados como novos casos cursaram para cura, já os que abandonaram o tratamento tiveram como desfecho o óbito. Verifica-se que a taxa mais alta de resistência medicamentosa estão relacionadas aos casos tratados 
anteriormente quando comparados com novos casos, nesse ponto é possível considerar que um caso novo tenha menos probabilidade de desenvolver resistências, já a maioria dos casos que foram interrompidos ou descontinuaram obtiveram os piores desfechos clínicos (MESFIN EA, et al., 2018)

Estudo conduzido na China constatou uma alta resistência à ofloxacina facilmente disponíveis no mercado ainda amplamente utilizado no tratamento anti-TB e geralmente empregado para infecções bacterianas respiratórias não diagnosticadas, o que pode justificar como provável motivo para resistência medicamentosa. Assim, diante dessas experiências entre diferentes pesquisas, percebe-se a importância do rigoroso controle no uso do esquema terapêutico que tem influência no surgimento de resistências e consequentemente no melhor ou pior desfecho do caso TB-DR (YAO S, et al., 2020).

Por fim, chama atenção informações ignoradas ou inconsistentes de algumas variáveis, o que pode ser considerado uma limitação do estudo. A incompletude ou subnotificação de dados se configura como um desafio para a caracterização do perfil dos pacientes, bem como o controle da doença (BARTHOLOMAY P, 2020).

De modo geral, é imprescindível considerar as medidas necessárias na intensificação da adequada notificação dos casos, dado que a partir do registro consegue-se visualizar o quadro situacional da doença. Desse modo, permitem-se ações assertivas na tomada de decisão e planejamento estratégico dos gestores e profissionais de saúde, que repercutirá na identificação real das necessidades dos indivíduos acometidos pela TB-DR, prestação da assistência de saúde com impacto no controle da infecção, aumento na adesão terapêutica e melhor qualidade de vida dos pacientes. Propõe-se, então, sensibilização dos profissionais na perspectiva de propiciar melhor comunicação no preenchimento dos documentos de registro e ficha de notificação relacionada aos casos de TB-DR.

Além disso, o estudo reflete a realidade de apenas uma referência terciária no atendimento aos pacientes com TB-DR, o que limita a generalização dos achados, assim como o pequeno número de casos TB-DR incluídos na amostra, contudo, destaca-se que o serviço hospitalar é referência estadual, recebendo pacientes de toda região metropolitana, como do interior.

\section{CONCLUSÃO}

Torna-se notória a importância em torno da adoção de estratégia para que haja o aumento da adesão terapêutica, como o TDO e o compartilhamento do cuidado entre Atenção Primária de Saúde e a Atenção Terciária. Nessa perspectiva, verifica-se a incompletude dos dados fornecidos pelo sistema de informação, o que traz a reflexão acerca da necessidade do aprimoramento nos serviços de Vigilância Epidemiológica. Vêse que tal problemática carrega consigo a possibilidade de repercutir negativamente no diagnóstico situacional, bem como nas estratégias para prevenção, controle e acompanhamento dos casos TB-DR.

\section{REFERÊNCIAS}

1. BALLESTERO JGA, et al. Estratégias de controle e atenção à tuberculose multirresistente: uma revisão da literatura. Rev Panam Salud Publica, 2019; 43:e20. Disponível em: https://www.ncbi.nlm.nih.gov/pmc/articles/PMC6459353/pdf/rpsp-43-e20.pdf.

2. BARTHOLOMAY P. Lacunas na vigilância da tuberculose drogaresistente: relacionando sistemas de informação no Brasil. Cad. Saúde Pública, 2020; 36(5): e00082219. Disponível em: https://www.scielosp.org/pdf/csp/2020.v36n5/e00082219/pt.

3. BRASIL. Ministério da Saúde. Secretaria de Vigilância em Saúde. Departamento de Vigilância das Doenças Transmissíveis. Manual de Recomendações para o Controle da Tuberculose no Brasil / Ministério da Saúde. Brasília: Ministério da Saúde; $2019 . \quad$ Disponível em: https://sbpt.org.br/portal/wpcontent/uploads/2019/06/manual_recomendacoes_tb_2ed_atualizada_8maio19.pdf.

4. BRASIL. Departamento de vigilância das doenças transmissíveis, Secretaria de Vigilância em Saúde. Ministério da Saúde. Guia de vigilância em saúde. Brasília: Ministério da Saúde, 2017.

5. BRASIL. Secretaria de Atenção à Saúde. Departamento de Ações Programáticas Estratégicas. Guia do pré-natal do parceiro para profissionais de Saúde, 2018a. Disponível em: https://portalarquivos2.saude.gov.br/images/pdf/2018/agosto/22/PNP.pdf.

6. BRASIL. Ministério da Saúde. Secretaria de Atenção à Saúde. Departamento de Ações Programáticas Estratégicas. Perfil da morbimortalidade masculina no Brasil, 2018b. Disponível em: https://www.saude.gov.br/images/pdf/2018/novembro/07/Perfil-da-morbimortalidade-masculina-no-Brasil.pdf. 
7. CAMINERO JA, et al. Diagnosisand Treatment of Drug-Resistant Tuberculosis. Diagnóstico y tratamiento de La tuberculosis com resistencia a fármacos. Arch Bronco neumol., 2017; 54(9): 501-509. doi: 10.1016/j.arbres.2017.02.006.

8. FERREIRA DP, et al. Prevalência da Coinfecção Hiv/Tb em Pacientes de um Hospital de Referência na Cidade do Rio De Janeiro. Rev Fund Care Online, 2019; 11(n. esp): 358-362. DOI: 10.9789/2175-5361. 2019.v11i2.358-362.

9. HO J. et al. Decentralized care for multidrug-resistant tuberculosis: a systematic review and meta-analysis. Bull World Health Organ, 2017; 95: 584-593. Disponível em: https://www.who.int/bulletin/volumes/95/8/17-193375.pdf.

10. GLASAUER S, et al. First-line tuberculosis drug resistance patternsand associated risk factors in Germany, $2008-2017$. PLoSOne, 2019; 14(6): e0217597. doi:10.1371/journal.pone.0217597.

11. GUPTA-WRIGHT A, et al. World TB day 2018: The challenge of drug resistant tuberculosis [version 1; referees: not peer reviewed. F 1000 Research, 2018; 7:217, 2018. Disponível em: https://www.ncbi.nlm.nih.gov/pmc/articles/PMC5824327/pdf/f1000research-7-15324.pdf.

12. NUGRAHAENI DN, ZAQIYA S. The relation ship between previous tuberculosis treatment and HIV status with multidrug-resistant tuberculosis. Jurnal Kese hatan Masyarakat, 2019; 14(3): 347-352. Disponível em: https://journal.unnes.ac.id/nju/index.php/kemas/article/view/14087/9205.

13. MESFIN EA, et al. Padrões de resistência a drogas de cepas de Mycobacterium tuberculosis e fatores de risco associados entre pacientes suspeitos de tuberculose multirresistentes da Etiópia. PLoSOne, 2018; 13(6): e0197737. doi: $10.1371 /$ journal.pone.0197737.

14. MORAES MFV, et al. Perfil epidemiológico de casos de tuberculose em um município prioritário no estado do maranhão. Ver Pesq Saúde, 2017; 18(3): 147-150.

15. OLIVEIRA LB, et al. Epidemiological analysis of tuberculosis/HIV coinfection. Cogitare Enferm., 2018; 23(1): e51016.2018.

16. OLIVEIRA SAG, et al. Adesão e qualidade de vida em pacientes com tuberculose pulmonar. Rev. enferm UFPE online, 2019; 13(3): 691-706. Disponível em: https://periodicos.ufpe.br/revistas/revistaenfermagem/article/view/236594/31564.

17. PARIDA A, et al. Comparison of Comparison of Directly Observed Treatment Short Course (DOTS) with SelfAdministered Therapy in Pulmonary Tuberculosis in Udupi District of Southern India. J Clin Diagn Res., 2014; 8(8): HC29-31.

18. RABAHI et al. Tratamento da tuberculose. J Bras Pneumol., 2017; 43(5): 472-486. Disponível em: https://www.scielo.br/pdf/jbpneu/v43n6/pt_1806-3713-jbpneu-43-06-00472.pdf.

19. SANTOS ML, et al. Fatores associados à subnotificação de tuberculose com base no Sinan Aids e Sinan Tuberculose. Rev. bras. epidemiol., 2018; 21: e180019.

20. SANTOS TA, MARTINS MMF. Perfil dos casos de reingresso após abandono do tratamento da tuberculose em Salvador, Bahia, Brasil.Cad. saúde colet., 2018; 26(3): 233-240.

21. SOUSA GJB, et al. Padrão temporal da cura, mortalidade e abandono do tratamento da tuberculose em capitais brasileiras. Rev. Latino-Am. Enfermagem, Ribeirão Preto, 27: 2019; e3218. Availablefrom: http://dx.doi.org/10.1590/1518-8345.3019.3218.

22. VIANA PVS, et al. Fatores associados ao abandon e ao óbito de casos de tuberculose drogarresistente (TBDR) atendidos em um centro de referência no Rio de Janeiro, Brasil. Cad. Saúde Pública, 2018; 34(5): e0004817. Disponível em: https://www.scielosp.org/pdf/csp/2018.v34n5/e00048217/pt.

23. World Health Organization. Global tuberculosis report 2017; [about 2 screens]. Available from: http://www.who.int/tb/publications/global_report/en/.

24. YAO S, et al. Determinação da resistência a medicamentos e fatores de risco para Mycobacterium tuberculosis para tuberculose multirresistente em pacientes ambulatoriais com tuberculose positiva para escarro na província de Anhui, China, 2015-2016. Infecção e resistência a medicamentos, 2020; 13: 1023-1032. doi: 10.2147/IDR.S244482. 